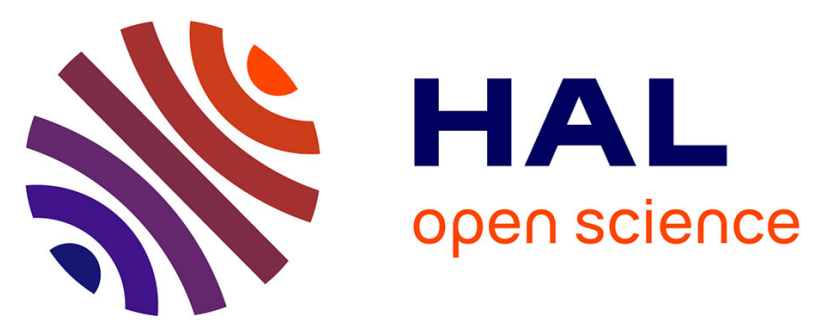

\title{
An Incremental formulation for the linear analysis of viscoelastic beams: Relaxation differential approach using generalized variables
}

\author{
Claude Chazal, Rostand Moutou Pitti, Alaa Chateauneuf
}

\section{- To cite this version:}

Claude Chazal, Rostand Moutou Pitti, Alaa Chateauneuf. An Incremental formulation for the linear analysis of viscoelastic beams: Relaxation differential approach using generalized variables. Mechanics of Time-Dependent Materials and Processes in Conventional and Multifunctional Materials,Proceedings of the 2011 Annual Conference on Experimental and Applied Mechanics, 3, pp 231-238, 2011, 10.1007/978-1-4614-0213-8_33 . hal-01616926

\section{HAL Id: hal-01616926 \\ https://hal.science/hal-01616926}

Submitted on 15 Oct 2017

HAL is a multi-disciplinary open access archive for the deposit and dissemination of scientific research documents, whether they are published or not. The documents may come from teaching and research institutions in France or abroad, or from public or private research centers.
L'archive ouverte pluridisciplinaire HAL, est destinée au dépôt et à la diffusion de documents scientifiques de niveau recherche, publiés ou non, émanant des établissements d'enseignement et de recherche français ou étrangers, des laboratoires publics ou privés. 


\title{
An Incremental formulation for the linear analysis of viscoelastic beams: Relaxation differential approach using generalized variables
}

\author{
Claude CHAZAL*, Rostand Mouto PITTI** and Alaa CHATEAUNEUF** \\ * Heterogeneous Material Research Group, Civil Engineering and Durability Team, Limoges \\ University, 19300 Egletons, FRANCE chazal@unilim.fr
}

** Clermont Université, Université Blaise Pascal, Laboratoire de Mécanique et Ingénieries, EA 3867, BP 206, 63000 Clermont Ferrand, FRANCE

\begin{abstract}
This paper is concerned with the development of a new incremental formulation in the time domain for linear, non-aging viscoelastic materials undergoing mechanical deformation. The formulation is derived from linear differential equations based on a discrete spectrum representation for the relaxation function. The incremental constitutive equations are then obtained by finite difference integration. Thus the difficulty of retaining the stress history in computer solutions is avoided. The influence of the whole past history on the behaviour at any time is given by a pseudo second order tensor. A complete general formulation of linear viscoelastic stress analysis is developed in terms of increments of midsurface strains and curvatures and corresponding stress resultants. The generality allowed by this approach has been established by finding incremental formulation through simple choices of the tensor relaxation components. This approach appears to open a wide horizon (to explore) of new incremental formulations according to particular relaxation components. Remarkable incremental constitutive laws, for which the above technique is applied, are given. This formulation is introduced in a finite element discretization in order to resolve complex boundary viscoelastic problems.
\end{abstract}

Keywords: Incremental formulation; Viscoelasticity; Discrete relaxation function; Generalized variables.

\section{Introduction}

Viscoelastic materials are characterized by possessing infinite memory. Their actual mechanical response is a function of the whole past history of stress and strain. In most cases, the behaviour of any linear viscoelastic material may be represented by a hereditary approach based on the superposition principle of Boltzmann. This implies that stress and strain analysis of viscoelastic phenomena which can be observed in the behaviour of many real materials, presents many difficulties for real problems of complex geometry. The important use of viscoelastic materials in civil engineering structures requires understanding the behaviour of time dependent mechanics fields which can be lead to collapse of such structures. The main problem in computation mechanics is to know the response of viscoelastic materials taking into account its complete past history of stress and strain. An intensive research work is 
investigated by a number of authors. Bozza and Gentili [1] use the theory of linear viscoelasticity to establish constitutive equations using relaxation functions. They seek solutions to the inversion problem of the constitutive equations. Aleksey Drozdov and AlDorfmann [2] derive constitutive equations for the nonlinear viscoelastic behaviour after performing tensile relaxation tests. Most of analytical solutions proposed in the literature can not deal with real and complex problems because these methods require the retaining of the complete past history of stress and strain in the memory of a digital computer.

In this context, a number of theories have been proposed in the past in order to formulate incremental constitutive equations for linear viscoelastic behaviour. Among them, Kim and Lee [3] and Theocaris [4] have proposed incremental formulation and constitutive equations in the finite element context (see also Chazal and Pitti [5]). In fracture viscoelastic mechanics, Moutou Pitti et al. [6-7] have applied the incremental formulation in order to evaluate creep crack growth process in viscoelastic media. Krempl [8] and Kujawski et al. [9] have performed experimental study of creep and relaxation in steel at room temperature. Also, Godunov et al. [10] and Duffrène et al. [11] have proposed relaxation viscoelastic models in order to traduce the behaviour of the material. However, the formulation used is based on the spectral decomposition using a generalized Maxwell model. To avoid the use of generalized Maxwell model, we will develop in this paper an alternative incremental formulation. This new formulation is based on discrete relaxation spectrum and the finite difference method using generalized differential equations in the time domain. The incremental stress and strain constitutive equations are not restricted to isotropic materials and can be used to resolve complex boundary viscoelastic problems without retaining the past history of the material in the memory of a digital computer.

First, we recall the discrete relaxation spectrum representation and its use in Boltzmann's superposition principle [12]. The one dimensional linear viscoelastic behaviour is used to account for three dimensional responses. After that, we present the development of the generalized differential equations in terms of one dimensional stress and strain components. Finally, the incremental viscoelastic constitutive equations of the model are established.

\section{Relaxation spectrum representation}

In this work, we will consider only small strains. According to the results obtained by Christensen [13], Mandel [14], Salençon [15] and Chazal and Dubois [16], the components of the relaxation tensor $\mathbf{G}(t)$ can be represented in terms of exponential series:

$G(t)=\left\{G^{\infty}+\sum_{\rho=1}^{R} G^{\rho} e^{-t \mu^{\rho}}\right\} H(t)$

where $\eta^{\rho}, \rho=1, \ldots, R$, are strictly positive scalars and repeated indices do not imply summation convention. $G^{\infty}$ and $G^{\rho}$ represent the equilibrium and the differed part of the relaxation function respectively and $H(t)$ is the Heaviside unit step function.

According to Boltzmann's principle superposition in linear non-ageing viscoelasticity [12], the constitutive equations between the component $\sigma(t)$ of the stress and the component of the strain $e(t)$ 
for non-ageing linear viscoelastic materials can be expressed in the time domain by the hereditary Volterra's integral equation:

$\sigma(t)=\int_{-\infty}^{t} G(t-\tau) \frac{\partial e_{\gamma \delta}(\tau)}{\partial \tau} d \tau$

We introduce stresses and strains in generalized variables according to Love's first-order shell theory. The strain at any point of the beam may be given as

$e(t)=\varepsilon(t)+\zeta \chi(t)$

where $\varepsilon(t)$ and $\chi(t)$ are the middle surface extensional strain and curvature, respectively. If we consider a plane stress state, the non-vanishing resultant of stresses is then defined by

$N(t)=\int_{-h / 2}^{+h / 2} \sigma(\zeta, t) d \zeta, M(t)=\int_{-h / 2}^{+h / 2} \zeta \sigma(\zeta, t) d \zeta$

$N(t)$ and $M(t)$ are the generalized stresses and $h$ is the thickness of the beam assumed to be constant. In order to determine the constitutive equation in terms of generalized stresses and strains, we introduce generalized strains, given by equation (3), into equation (2). One find

$\sigma^{N}(t)+\sigma^{M}(t)=\int_{-\infty}^{t} G(t-\tau) \frac{\partial}{\partial \tau}[\varepsilon(\tau)+\zeta \chi(\tau)] d \tau$

Note that the total stress $\sigma(t)$ is separated into two parts: normal stress $\sigma^{N}(t)$ due to extensional strain and bending stress $\sigma^{M}(t)$ due to curvature. The constitutive equations in generalized variables can now be obtained from behaviour equation (5).

Using equation (4) and integrating equation (5) over the thickness, we find

$$
\begin{aligned}
& N(t)=\int_{-h / 2}^{+h / 2} \sigma^{N}(\zeta, t) d \zeta=h \int_{-\infty}^{t} G(t-\tau) \frac{\partial}{\partial \tau} \varepsilon(\tau) d \tau \\
& M(t)=\int_{-h / 2}^{+h / 2} \zeta \sigma^{M}(\zeta, t) d \zeta=\frac{h^{3}}{12} \int_{-\infty}^{t} G(t-\tau) \frac{\partial}{\partial \tau} \chi(\tau) d \tau
\end{aligned}
$$

\section{Formulation of differential equations}

When we apply the mechanical strain defined by the strain history $\{e(\tau), \tau \in \mathfrak{R}\}$, the response of the material is then given by the history of stresses $\{N(t), \mathrm{M}(t), t \in \mathfrak{R}\}$ defined by the behaviour equations $(6,7)$ in which the relaxation function is given by equation (1).

If the generalized $\operatorname{strain}\{\varepsilon(t), \chi(t)\}$ is applied to the material at time $t$, then the response in stresses can be obtained using the finite relaxation spectrum representation given by equation (1). This leads to

$$
\left\{\begin{array}{l}
N(t) \\
M(t)
\end{array}\right\}=\int_{-\infty}^{t}\left[G^{\infty}+\sum_{\rho=1}^{R} G^{\rho} e^{-\mu^{\rho}(t-\tau)}\right] \frac{\partial}{\partial \tau}\left\{\begin{array}{c}
h \varepsilon(\tau) \\
\frac{h^{3}}{12} \chi(\tau)
\end{array}\right\}
$$


Thus the stresses given by the last equation, and written as a function of equilibrium and differed part of the relaxation spectrum, can be rewritten in the following form

$$
\left\{\begin{array}{l}
N(t) \\
M(t)
\end{array}\right\}=\left\{\begin{array}{c}
N^{\infty}(t)+\sum_{\rho=1}^{R} N^{\rho}(t) \\
M^{\infty}(t)+\sum_{\rho=1}^{R} M^{\rho}(t)
\end{array}\right\}
$$

with

$$
\begin{aligned}
& N^{\infty}(t)=\int_{-\infty}^{t} h \mathrm{G}^{\infty} \frac{\partial \varepsilon(\tau)}{\partial \tau} d \tau=h \mathrm{G}^{\infty} \varepsilon(t) \\
& N^{\rho}(t)=\int_{-\infty}^{t} h \mathrm{G}^{\rho} e^{-\mu^{\rho}(t-\tau)} \frac{\partial \varepsilon(\tau)}{\partial \tau} d \tau \\
& M^{\infty}(t)=\int_{-\infty}^{t} \frac{h^{3}}{12} \mathrm{G}^{\infty} \frac{\partial \chi(\tau)}{\partial \tau} d \tau=\frac{h^{3}}{12} \mathrm{G}^{\infty} \chi(t) \\
& M^{\rho}(t)=\int_{-\infty}^{t} \frac{h^{3}}{12} \mathrm{G}^{\rho} e^{-\mu^{\rho}(t-\tau)} \frac{\partial \chi(\tau)}{\partial \tau} d \tau
\end{aligned}
$$

It should be noted that $N^{\infty}(t)$ and $M^{\infty}(t)$ represent the equilibrium part of the mono-dimensional stress of the material while $N^{\rho}(t)$ and $M^{\rho}(t)$ represent the differed part of the same mono-dimensional stress.

As we mentioned in the above section, a differential approach is used in order to establish the differential equations of the mechanical model. Thus, we need to express the viscoelastic response of the material as a function of stress and strain derivatives. For this reason, let us use equation (9), the rate of the total stress is determined by

$$
\frac{\partial}{\partial t}\left\{\begin{array}{l}
N(t) \\
M(t)
\end{array}\right\}=\frac{\partial}{\partial t}\left\{\begin{array}{c}
N^{\infty}(t)+\sum_{\rho=1}^{R} N^{\rho}(t) \\
M^{\infty}(t)+\sum_{\rho=1}^{R} M^{\rho}(t)
\end{array}\right\}
$$

The rate of the equilibrium part of the one-dimensional stress $N^{\infty}(t)$ and $M^{\infty}(t)$ is easy to be evaluated. According to equations (10a) and (10c), and after applying a time derivative operator, one find

$$
\begin{aligned}
& \frac{\partial N^{\infty}(t)}{\partial t}=h \mathrm{G}^{\infty} \frac{\partial \varepsilon(t)}{\partial \mathrm{t}} \\
& \frac{\partial M^{\infty}(t)}{\partial t}=\frac{h^{3}}{12} \mathrm{G}^{\infty} \frac{\partial \chi(t)}{\partial \mathrm{t}}
\end{aligned}
$$


In other words, the equilibrium part of the one-dimensional stress is directly proportional to the total strain at time $t$. It is completely defined by the history of the applied strain. However, the rate of the differed part of the one-dimensional stresses $N^{\rho}(t)$ and $M^{\rho}(t)$ is more complicated to be determined. Using equations (10b) and (10d), and applying a time derivative operator, we can write

$$
\begin{aligned}
& \frac{1}{h} \frac{\partial}{\partial t} N^{\rho}(t)=G^{\rho} e^{-\mu^{\rho}(t-t)} \frac{\partial}{\partial t} \varepsilon(t)-\int_{-\infty}^{t} G^{\rho} \mu^{\rho} e^{-\mu^{\rho}(t-\tau)} \frac{\partial}{\partial \tau} \varepsilon(\tau) \\
& \frac{12}{h^{3}} \frac{\partial}{\partial t} M^{\rho}(t)=G^{\rho} e^{-\mu^{\rho}(t-t)} \frac{\partial}{\partial t} \chi(t)-\int_{-\infty}^{t} G^{\rho} \mu^{\rho} e^{-\mu^{\rho}(t-\tau)} \frac{\partial}{\partial \tau} \chi(\tau)
\end{aligned}
$$

These integral equations give the total rate of the differed part of the mono-dimensional stresses.

The main purpose of our development in this section is to establish differential equations between the total rate of the mono-dimensional stresses and the total strain. For this reason, we will transform last equations in differential type.

Let us introduce the behaviour equations (10b) and (10d) in the integral equations (13). This leads to linear differential equations with constant coefficients and can be integrated analytically

$$
\begin{aligned}
& \frac{\partial}{\partial t} N^{\rho}(t)+\mu^{\rho} \mathrm{N}^{\rho}(t)=h \mathrm{G}^{\rho} \frac{\partial}{\partial t} \varepsilon(t) \\
& \frac{\partial}{\partial t} M^{\rho}(t)+\mu^{\rho} \mathrm{M}^{\rho}(t)=\frac{h^{3}}{12} \mathrm{G}^{\rho} \frac{\partial}{\partial t} \chi(t)
\end{aligned}
$$

The solution of these linear differential equations gives the rate of the one-dimensional stresses $N^{\rho}(t)$ and $M^{\rho}(t)$.

It should be mentioned that the non-ageing linear viscoelastic behaviour is completely defined by the differential equations (12) and (14). We note that this formulation, written in terms of generalized stresses and strains rates, is easily adapted to temporal discretization methods such as finite difference method.

\section{Incrementalization of the differential equations}

Here we will describe the solution process of a step-by-step nature in which loads are applied stepwise at various time intervals. Let us consider the time step $\Delta t_{n}=t_{n+1}-t_{n}$. The subscript $n$ and $n+1$ refer to the values at the beginning and end of time step, respectively. This technique is successfully used by Chazal and Dubois in [16] in the case of viscoelastic structures. We assume that the time derivative during each time increment is. A linear approximation is used for strains and is expressed by

$$
\left\{\begin{array}{l}
\varepsilon(\tau) \\
\chi(\tau)
\end{array}\right\}=\left\{\begin{array}{l}
\varepsilon\left(t_{n}\right) \\
\chi\left(t_{n}\right)
\end{array}\right\}+\frac{\tau-t_{n}}{\Delta t_{n}} H\left(\tau-t_{n}\right)\left\{\begin{array}{l}
\varepsilon\left(t_{n+1}\right)-\varepsilon\left(t_{n}\right) \\
\chi\left(t_{n+1}\right)-\chi\left(t_{n}\right)
\end{array}\right\}
$$


This linear approximation leads to very accuracy results in finite element discretization as it is shown by Chazal and Dubois [16]. Thus we don't need higher approximations for the strain during a finite increment of time load. This leads to a constant rate during each time increment:

$\frac{\partial}{\partial t}\left\{\begin{array}{l}\varepsilon\left(t_{n}\right) \\ \chi\left(t_{n}\right)\end{array}\right\}=\frac{1}{\Delta t_{n}}\left\{\begin{array}{l}\varepsilon\left(t_{n+1}\right)-\varepsilon\left(t_{n}\right) \\ \chi\left(t_{n+1}\right)-\chi\left(t_{n}\right)\end{array}\right\}=\frac{1}{\Delta t_{n}}\left\{\begin{array}{l}\Delta \varepsilon\left(t_{n}\right) \\ \Delta \chi\left(t_{n}\right)\end{array}\right\}$

By integrating equation (11) between $t_{n}$ and $t_{n+1}$, it can be written in the following form

$$
\left\{\begin{array}{c}
\Delta N\left(t_{n}\right) \\
\Delta M\left(t_{n}\right)
\end{array}\right\}=\sum_{\rho=1}^{R}\left\{\begin{array}{c}
\Delta N^{\rho}\left(t_{n}\right) \\
\Delta M^{\rho}\left(t_{n}\right)
\end{array}\right\}+G^{\infty}\left\{\begin{array}{c}
h \Delta \varepsilon\left(t_{n}\right) \\
\frac{h^{3}}{12} \Delta \chi\left(t_{n}\right)
\end{array}\right\}
$$

In order to determine the generalized stress increments from this equation, we have to determine the generalized pseudo stress increments $\Delta N^{\rho}\left(t_{n}\right)$ and $\Delta M^{\rho}\left(t_{n}\right)$ during the time step $\Delta t_{n}$. First, let us consider the differential equation (14). The analytical solution of this differential equation can be expressed as

$$
\begin{aligned}
& N^{\rho}\left(t_{n+1}\right)-N^{\rho}\left(t_{n}\right)=\left[e^{-\mu^{\rho} \Delta t_{n}}-1\right] N^{\rho}\left(t_{n}\right)+h \mathrm{G}^{\rho} \frac{\Delta \varepsilon\left(t_{n}\right)}{\mu^{\rho} \Delta t_{n}}\left[1-e^{-\mu^{\rho} \Delta t_{n}}\right] \\
& M^{\rho}\left(t_{n+1}\right)-M^{\rho}\left(t_{n}\right)=\left[e^{-\mu^{\rho} \Delta t_{n}}-1\right] M^{\rho}\left(t_{n}\right)+\frac{h^{3}}{12} \mathrm{G}^{\rho} \frac{\Delta \chi\left(t_{n}\right)}{\mu^{\rho} \Delta t_{n}}\left[1-e^{-\mu^{\rho} \Delta t_{n}}\right]
\end{aligned}
$$

The incremental constitutive equations can now be obtained from the constitutive equation (17). Substituting equations (18a) and (18b) into (17), we find

$$
\left\{\begin{array}{l}
\Delta N\left(t_{n}\right) \\
\Delta M\left(t_{n}\right)
\end{array}\right\}=\left[\begin{array}{cc}
\Pi\left(t_{n}\right) & 0 \\
0 & \Xi\left(t_{n}\right)
\end{array}\right]\left\{\begin{array}{l}
\Delta \varepsilon\left(t_{n}\right) \\
\Delta \chi\left(t_{n}\right)
\end{array}\right\}-\left\{\begin{array}{l}
\tilde{N}\left(t_{n}\right) \\
\tilde{M}\left(t_{n}\right)
\end{array}\right\}
$$

where $\Pi\left(t_{n}\right)$ and $\Xi\left(t_{n}\right)$ are scalar tensors which can be interpreted as rigidity tensors in extensional and bending state respectively, they are given by

$$
\begin{aligned}
& \Pi\left(t_{n}\right)=G^{\infty}+h \sum_{\rho=1}^{R} \frac{G^{\rho} \Delta \varepsilon\left(t_{n}\right)}{\mu^{\rho} \Delta t_{n}}\left[1-\mathrm{e}^{-\mu^{\rho} \Delta t_{n}}\right] \\
& \Xi\left(t_{n}\right)=G^{\infty}+\frac{h^{3}}{12} \sum_{\rho=1}^{R} \frac{G^{\rho} \Delta \chi\left(t_{n}\right)}{\mu^{\rho} \Delta t_{n}}\left[1-\mathrm{e}^{-\mu^{\rho} \Delta t_{n}}\right]
\end{aligned}
$$

$\tilde{N}\left(t_{n}\right)$ and $\tilde{M}\left(t_{n}\right)$ are pseudo generalized stresses which represent the influence of the complete past history of extensional and bending generalized stresses. They are given by 
$\left\{\begin{array}{c}\tilde{N}\left(t_{n}\right) \\ \tilde{M}\left(t_{n}\right)\end{array}\right\}=\sum_{\rho=1}^{R}\left[1-e^{-\mu^{\rho} \Delta t_{n}}\right]\left\{\begin{array}{l}N^{\rho}\left(t_{n}\right) \\ M^{\rho}\left(t_{n}\right)\end{array}\right\}$

The incremental constitutive law represented by equation (19) can be introduced in a finite element discretization in order to obtain solutions to complex viscoelastic problems.

Finally, in order to use the incremental viscoelastic formulation presented in this paper, we need to identify the relaxation components of the relaxation tensor. The experimental identification of viscoelastic properties is treated in details by Andreas and Lackner [17] and Herbert and Andreas [18]. The viscoelastic solution is obtained by the application of the method of functional equations to the elastic solution of the indentation problem and by means of torsional rheometry.

\section{Conclusions}

The transformation in differential terms of the integral formulation of the viscoelastic continuum problem has been successfully achieved through the introduction of a discrete spectrum representation of the relaxation function. This leads to a new linear incremental formulation in the time domain for nonageing viscoelastic materials undergoing mechanical deformation. The formulation is based on a differential approach using discrete spectrum representation for the relaxation components. The governing equations are then obtained using a discretized form of the Boltzmann's principle [12]. The analytical solution of the differential equations is obtained using finite difference discretization in the time domain. In this way, the incremental constitutive equations of linear viscoelastic material use a pseudo rigidity scalar tensor and the influence of the whole past history on the behaviour of the material at time $t$ is given by a pseudo first order tensor. The generality allowed by this approach has been established by finding incremental formulation through simple choices of the function relaxation components. This approach appears to open a wide horizon (to explore) of new incremental formulations according to particular relaxation components. Remarkable incremental constitutive laws, for which the above technique is applied, are given.

Among the numerous applications of the incremental formulations presented in this paper, is the numerical implementation in finite element software, thus the behaviour of complex boundary viscoelastic problems can be obtained.

\section{References}

1- Bozza A. and Gentili G. (1995) Inversion and Quasi-Static Problems in Linear Viscoelasticity. Meccanica 30:321-335

2- Aleksey D. Drozdov and AlDorfmann (2004) A Constitutive Model in Finite Viscoelasticity of Particle-reinforced Rubbers. Meccanica 39:245-270

3. Kim K.S, Sung LEE H (2007) An incremental formulation for the prediction of two-dimensional fatigue crack growth with curved paths. Int J Num Methods Eng 72:697-721

4. Theocaris P.S (1964) Creep and relaxation contraction ratio of linear viscoelastic materials. J Mech Physics Solids 12:125-138 
5. Chazal C, Moutou Pitti R (2009) An incremental constitutive law for ageing viscoelastic materials: a three dimensional approach. C. R. Mecanique 337:30-33

6. Mouto Pitti R, Chazal C, Labesse F, Lapusta Y (2011) A generalization of Mv integral to axisymmetric problems for viscoelastic materials, accepted for publication in Acta Mechanica, DOI 10.1007/s00707-011-0460-8

7. Moutou Pitti R, Chateauneuf A, Chazal C (2010) Fiabilité des structures en béton précontraint avec prise en compte du comportement viscoélastique, Fiabilité des Matériaux et des Structures, 6èmes Journées Nationales de Fiabilité Toulouse, France

8. Krempl E, (1979) An experimental study of uniaxial room-temperature rate-sensitivity, creep and relaxation of AISI type 304 stainless steel. J Mech Physics Solids 27:363-375.

9. Kujawski D, Kallianpur V, Krempl E (1980) An experimental study of uniaxial creep, cyclic Creep and relaxation of AISI type 304 stainless steel at room temperature. J Mech Physics Solids 28:129-148.

10. Godunov S.K, Denisenko V.V, Kozin N.S, Kuzmina N.K (1975) Use of relaxation viscoelastic model in calculating uniaxial homogeneous strains and refining the interpolation equations for maxwellian viscosity. J Appl Mech Tech Physics 16:811-814.

11. Duffrène L, Gy R, Burlet H, Piques R, Faivre A, Sekkat A, Perez J (1997) Generalized Maxwell model for the viscoelastic behavior of a soda-lime-silica glass under low frequency shear loading . Rheologica Acta, 36:173-186.

12. Boltzmann L (1878) Zur Theorie der elastischen Nachwirkung Sitzungsber, Mat Naturwiss. K1. Kaiser. Akad. Wiss 70, 275.

13. Christensen R.M (1971) Theory of Viscoelasticity: an Introduction. Academic Press, New York. ISBN 0-12-174250-4

14. Mandel J (1978) Dissipativité normale et variables caches. Mech Res Commun 5:225-229.

15. Salençon J (1983) Viscoélasticité. Presse de l'école nationale des ponts et chaussées, Paris.

16. Chazal C, Dubois F (2001) A new incremental formulation in the time domain of crack initiation in an orthotropic linearly viscoelastic solid. Mech Time-Depend Mater 5:229-253.

17. Andreas Jäger, Roman Lackner et al. (2007) Identification of viscoelastic properties by means of nanoindentation taking the real tip geometry into account. Meccanica 42:293-306

18- Herbert W. Müllner, Andreas Jäger et al. (2008) Experimental identification of viscoelastic properties of rubber compounds by means of torsional rhemetry. Meccanica 43:327-337 